Supplement of Hydrol. Earth Syst. Sci., 24, 4659-4674, 2020

https://doi.org/10.5194/hess-24-4659-2020-supplement

(c) Author(s) 2020. This work is distributed under

the Creative Commons Attribution 4.0 License.

(c) (1)

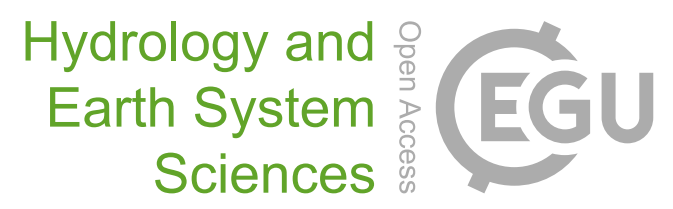

Supplement of

\title{
Estimation of subsurface soil moisture from surface soil moisture in cold mountainous areas
}

Jie Tian et al.

Correspondence to: Baoqing Zhang (baoqzhang@lzu.edu.cn) and Chansheng He (he@wmich.edu)

The copyright of individual parts of the supplement might differ from the CC BY 4.0 License. 


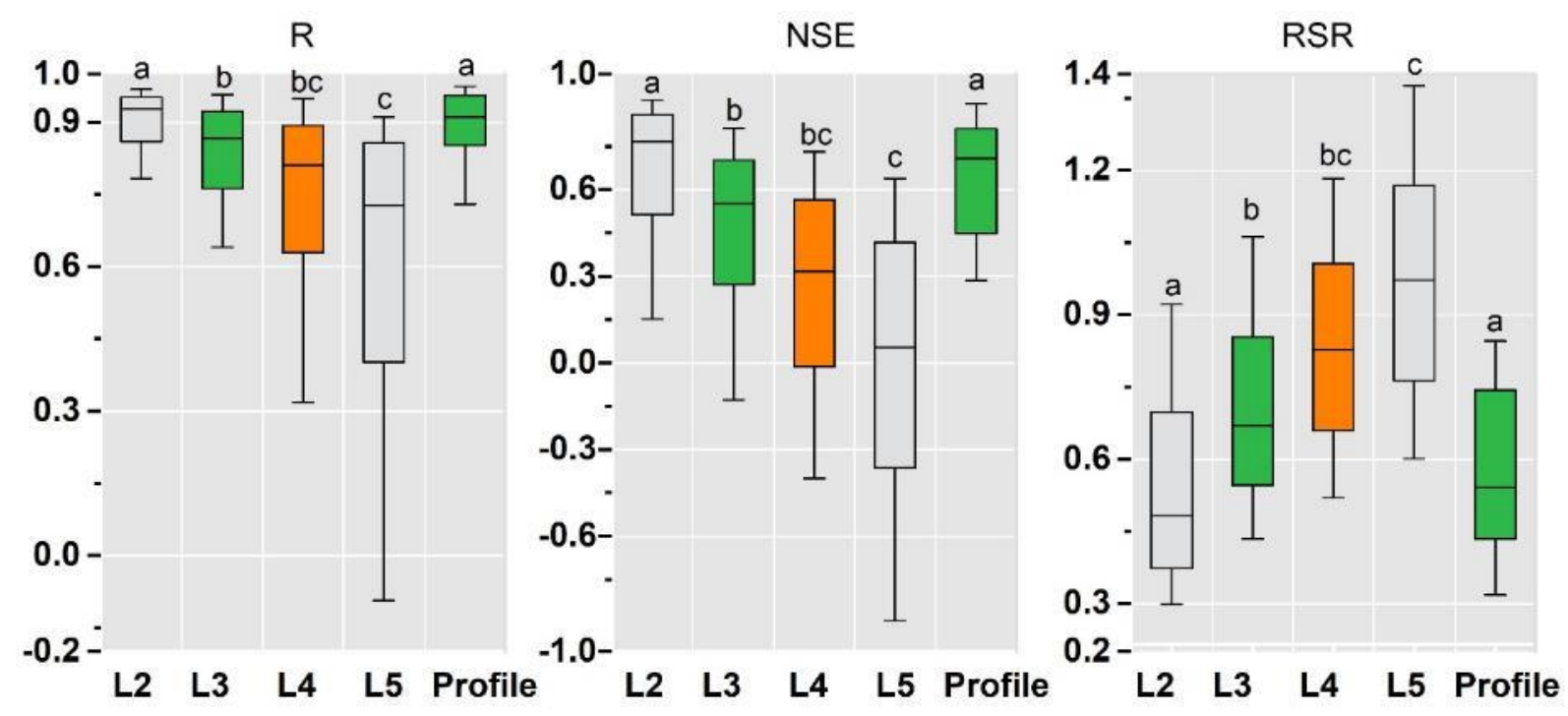

Fig. S1. Boxplot of the metrics (NSE, RSR, R) to evaluate the subsurface SM estimation using the surface SM by the ExpF method with the observations of the 35 stations during the growing seasons of 2014 to 2016 . Different letters above the box indicate the significant difference $(\mathrm{p}<0.05)$ among the different layers. 
0.4 D10: RMSE $=0.048, \mathrm{R}=0.45$

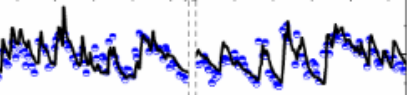

$\mathrm{D} 8: \mathrm{RMSE}=0.039, \mathrm{R}=0.57$

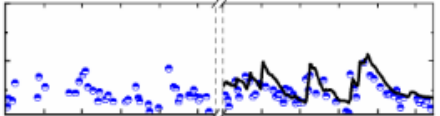

D11: $R M S E=0.033, R=0.69$

0.2
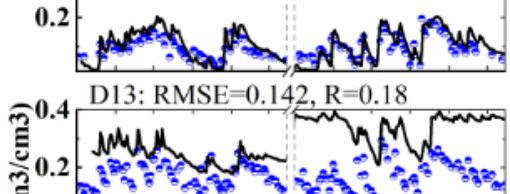

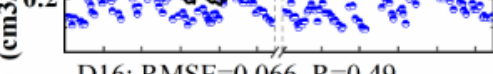

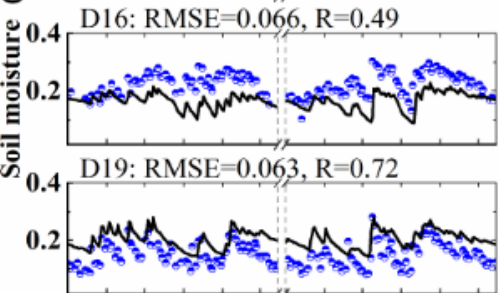

0.4 D22: $R M S E=0.043, R=0.48$

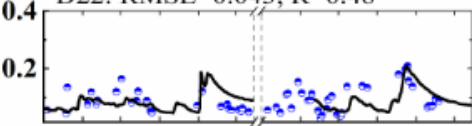

$0.4 \mathrm{D} 25: \mathrm{RMSE}=0.052, \mathrm{R}=0.68$

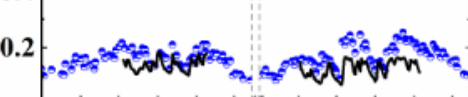
0.4 D28: RMSE $=0.051, \mathrm{R}=0.59$
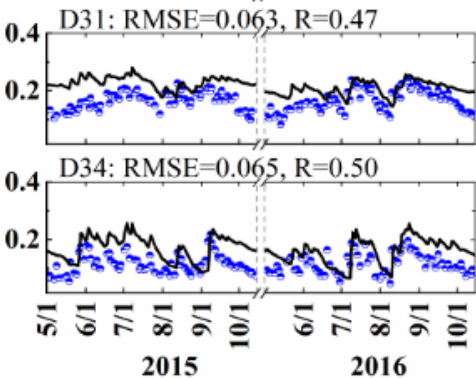

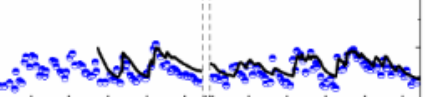

D14: $\mathrm{RMSE}=0.058, \mathrm{R}=0.73$

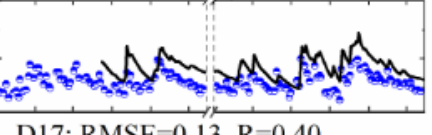

D17: $\mathrm{RMSE}=0.13, \mathrm{R}=0.40$

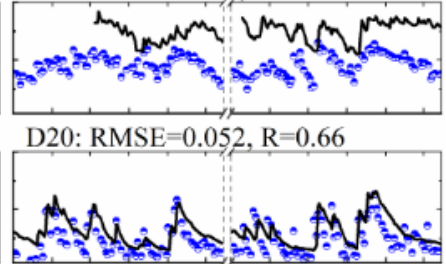

D23: $\mathrm{RMSE}=0.034, \mathrm{R}=0.53$

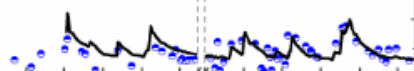

D26: $R M S E=0.026, R=0.67$

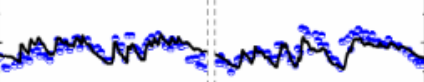

$\mathrm{D} 29: \mathrm{RMSE}=0.055, \mathrm{R}=0.73$
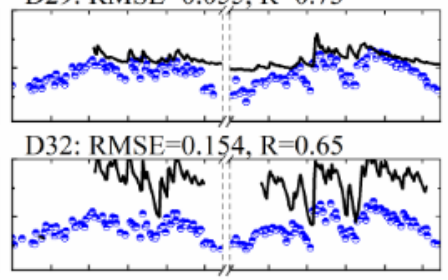

D35: $\mathrm{RMSE}=0.045, \mathrm{R}=0.31$
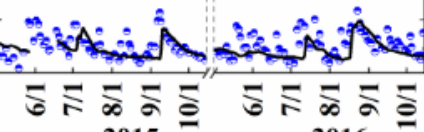

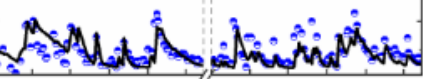

D6: $\mathrm{RMSE}=0.05,3, \mathrm{R}=0.58$

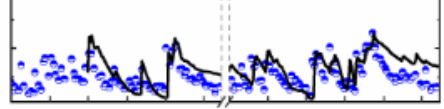

D9: $\mathrm{RMSE}=0.035, \mathrm{R}=0.66$

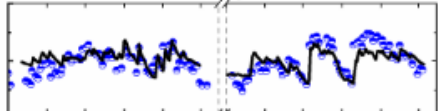

D12: $\mathrm{RMSE}=0.055, \mathrm{R}=0.69$
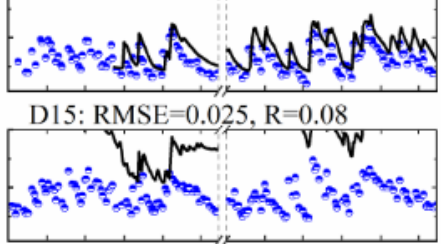

D18: $\mathrm{RMSE}=0.056, \mathrm{R}=0.52$

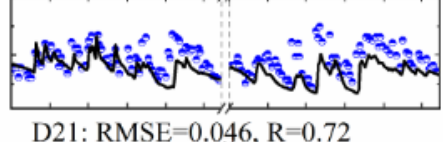

D21: $\mathrm{RMSE}=0.046, \mathrm{R}=0.72$

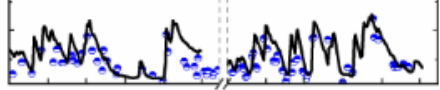

$\mathrm{D} 24: \mathrm{RMSE}=0.080, \mathrm{R}=0.58$

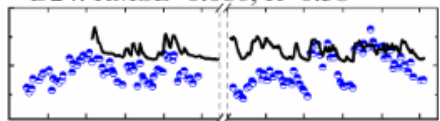

$\mathrm{D} 27: \mathrm{RMSE}=0.057, \mathrm{R}=0.39$

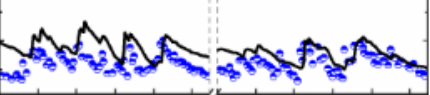

D30: $\mathrm{RMSE}=0.090, \mathrm{R}=0.73$

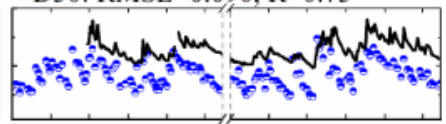

D33: $\mathrm{RMSE}=0.038, \mathrm{R}=0.36$

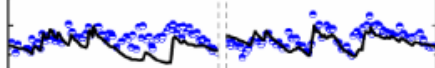

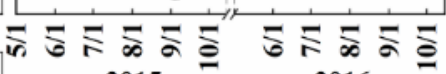

2015

2016

SMAP $\left(\mathrm{cm}^{3} / \mathrm{cm}^{3}\right)$

Observation $\left(\mathrm{cm}^{3} / \mathrm{cm}^{3}\right)$

Fig. S2. Comparison of the SMAP and in-situ observations of surface soil moisture $\left(\mathrm{cm}^{3} / \mathrm{cm}^{3}\right)$ data during the growing season of $2015-2016$ for the 35 stations. Also with the RMSE and R of the comparisons of the two datasets for each station during the growing season of 20152016. 
0.0 .

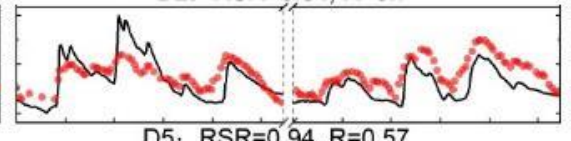

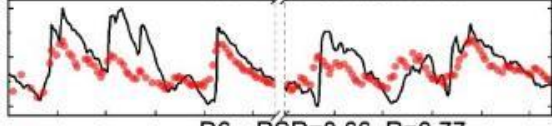

:

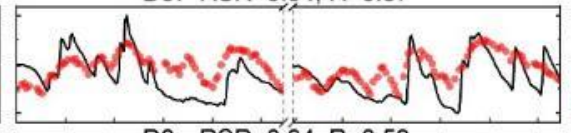
年 D8: RSR $=0.94, R=0.59$

D10: RSR=0.84, R=0.78

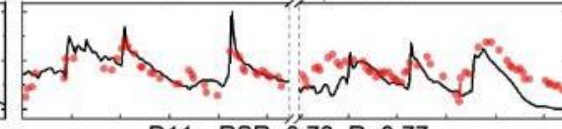
D11: $R S R=0,73, R=0.77$

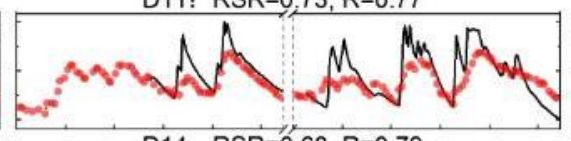

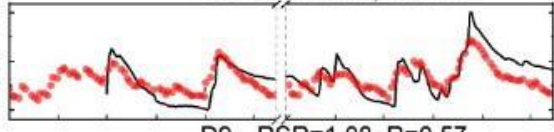
D9: $R S R=1.08, R=0.57$

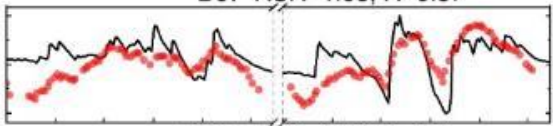

D12: $R S R=0.72, R=0.8$ D14: $R S R=0,68, R=0.79$
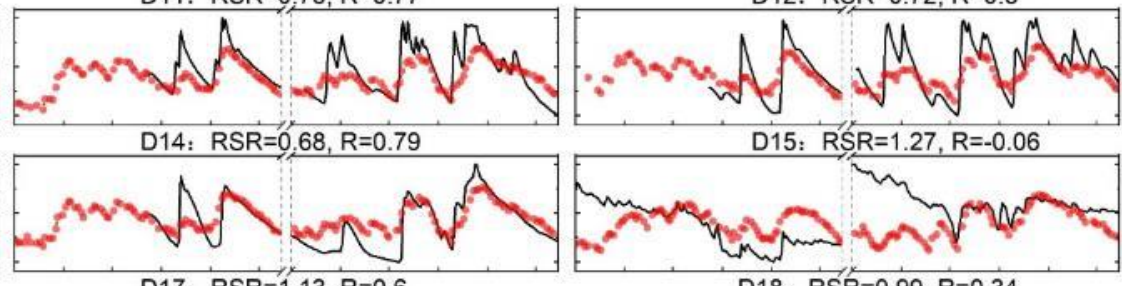

D15: $R S S=1.27, R=-0.06$
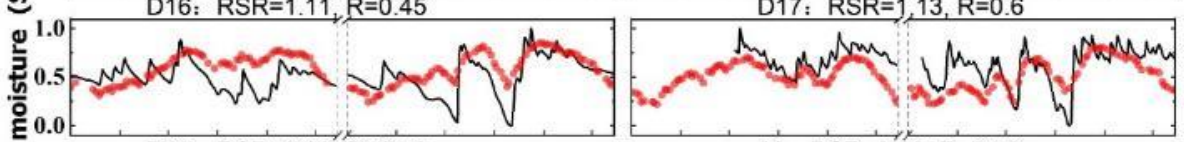

iे 1.0 D19: RSR $=0.83, R=0.78$

20: $R S R=0.62, R=0.79$

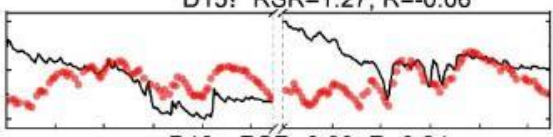

D18: $R S^{\prime}=0.99, R=0.34$

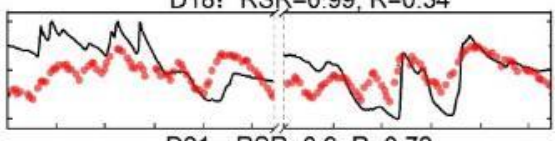

D21: $R S R=0.9, R=0.72$

0.5 (1.0)

D22: RSR=0.87, $R=0.58$

D25: $R S R=0.91, R=0.52$

D28: $R S R=0.88, R=0.68$
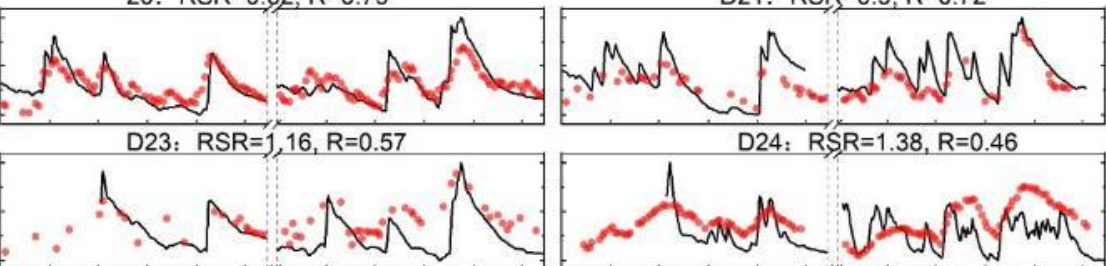

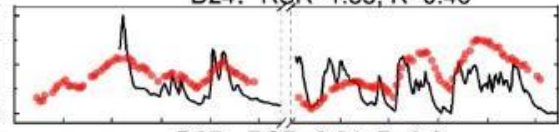

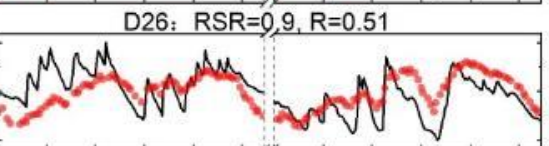

D31: $R S R=0.97, R=0.48$

D34: $R S R=0.82, R=0.57$

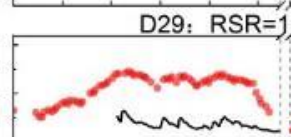

$1,69, R=0.61$

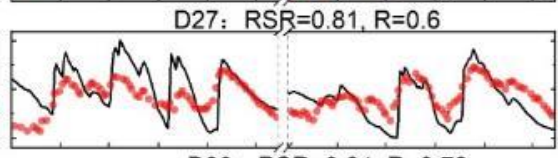

$D 30: R S R=0.84, R=0.72$
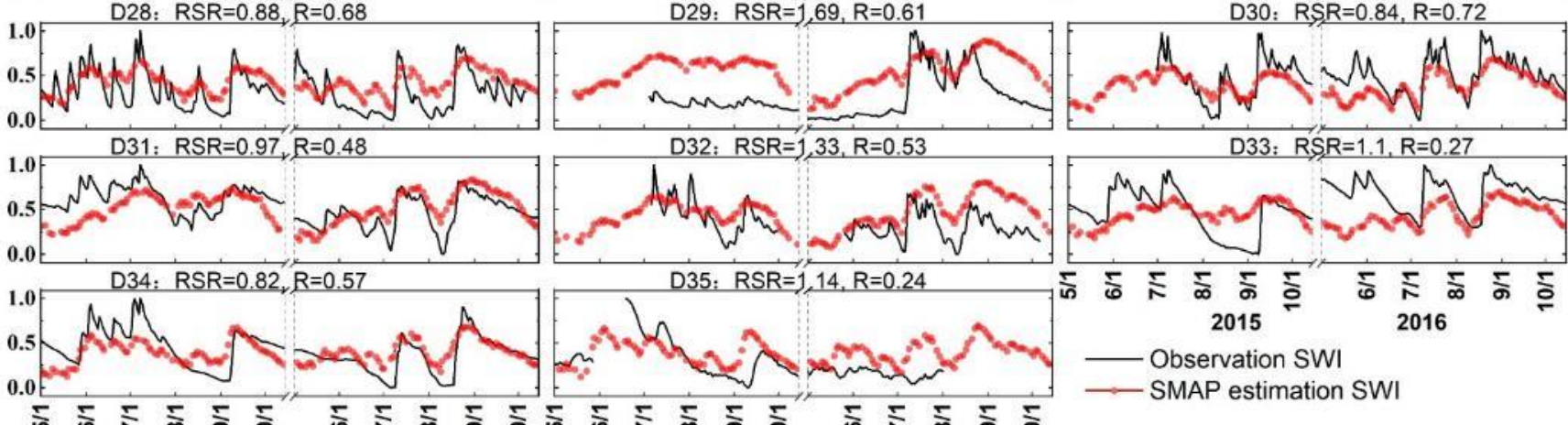

D33: $R S R=1.1, R=0.27$
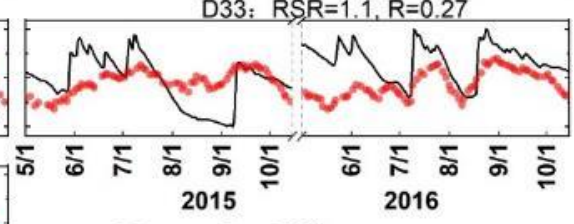

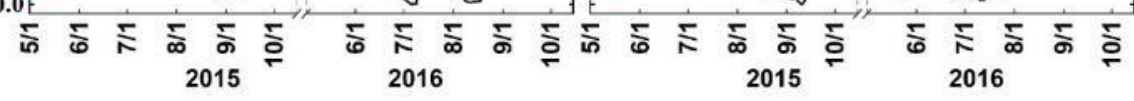

Observation SWI

SMAP estimation SWI

Fig. S3. Comparison of the SMAP estimation and in-situ observation of layer 2 SWI time series during the growing season of $2015-2016$ for the 35 stations. Also with the RMSE and R of the comparisons of the two datasets for each station during the growing season of 2015 2016 . 
D1: $R S R=1.29, R=-0.01$
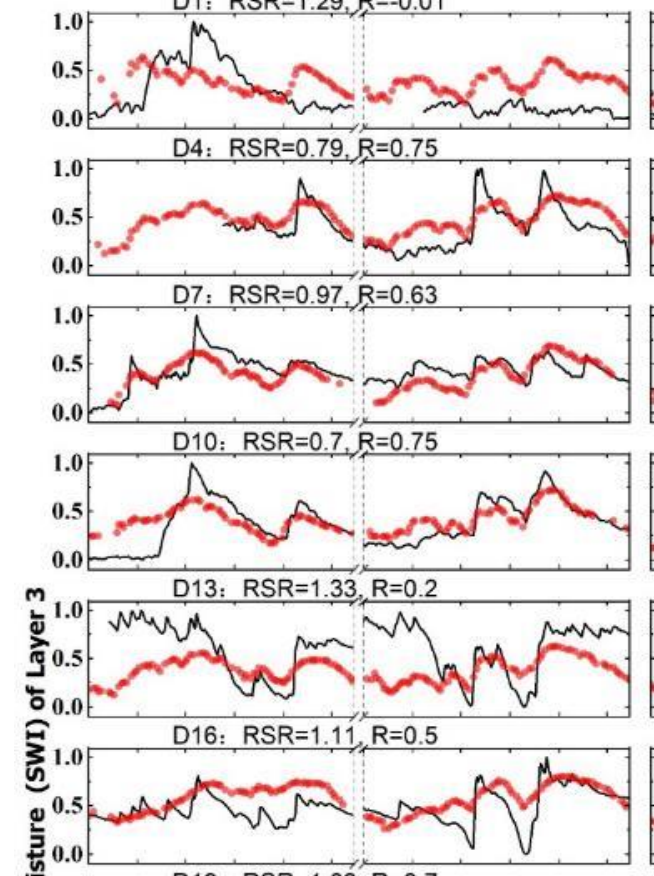

蒙 0.0

हुํำ
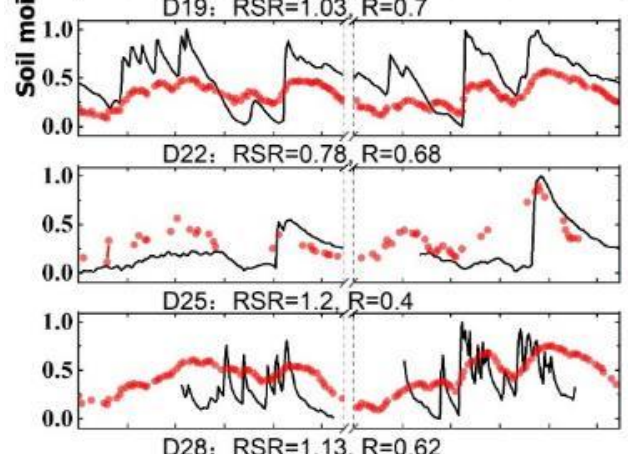

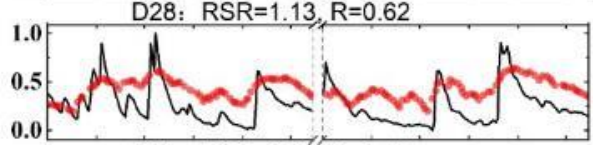

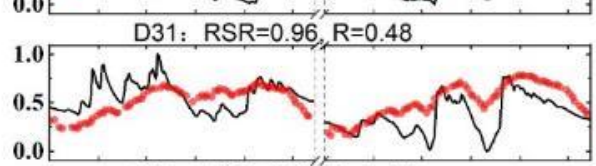

D34: $R S R=0.95, R=0.41$

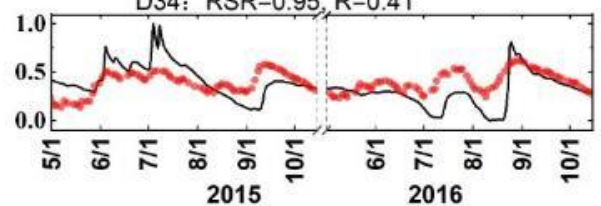

$\mathrm{D} 2: \mathrm{RS}, \mathrm{R}=1.23, \mathrm{R}=0.52$

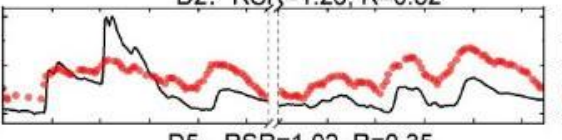

D5: $R S B=1.02, R=0.35$

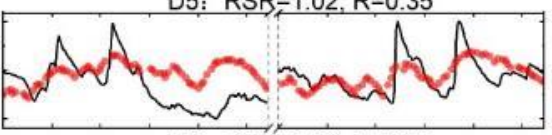

$D 8$ : $R S R=0.86, R=0.51$

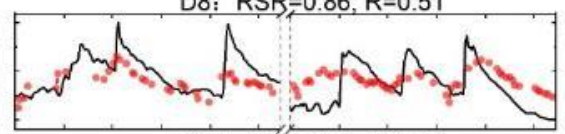

D11: $R S R=0.8, R=0.67$

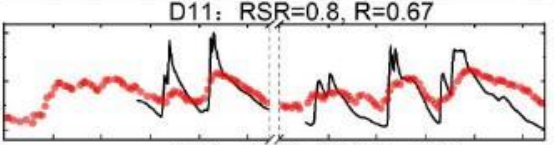

D14: $R S R=0.82, R=0.76$

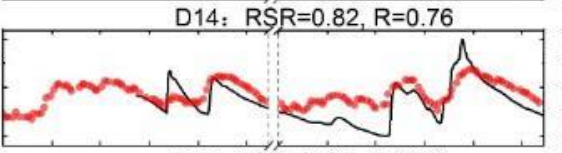

D17: $R S R=0.86, R=0.56$

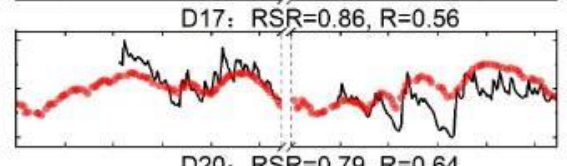

D20: $R S R=0.79, R=0.64$
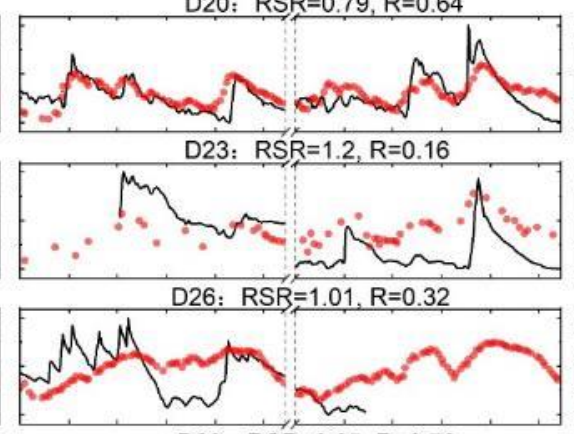

D29: $R S R=0.85, R=0.78$

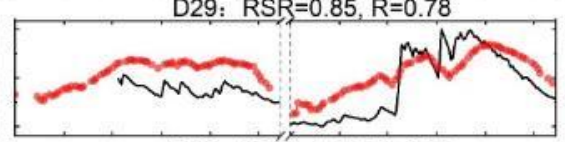

D32: $R S R=1.48, R=0.39$
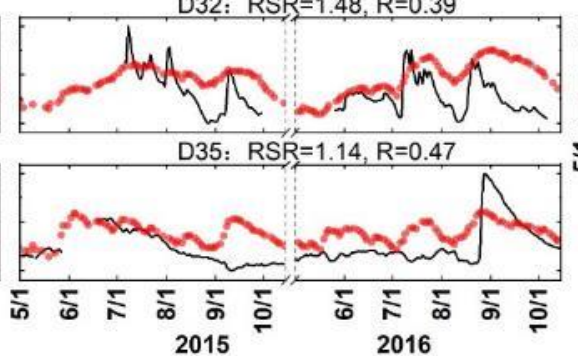

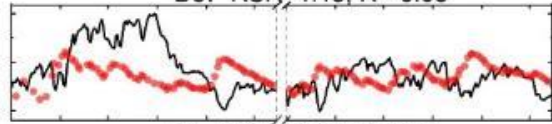

D6: $R S R=0.88, R=0.73$

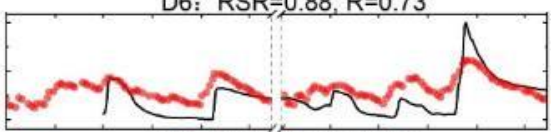

$\mathrm{D9}: \mathrm{RSR}=1.25, \mathrm{R}=0.21$

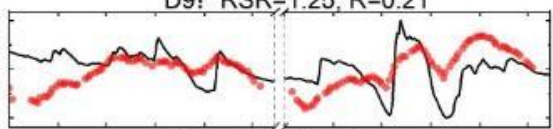

D12: $R S R=0.76, R=0.74$

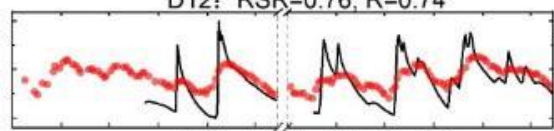

D15: $R S R=1.37, R=-0.3$

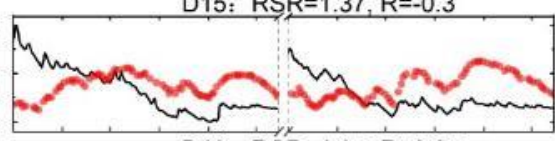

D18: $R S R=1.07, R=0.05$

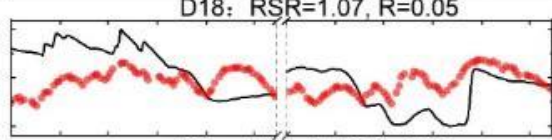

D21: $R S R=0.96, R=0.62$

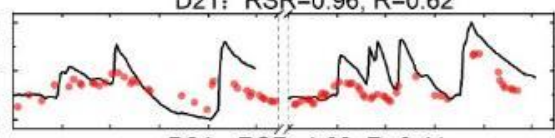

D24: $R S R=1.08, R=0.41$

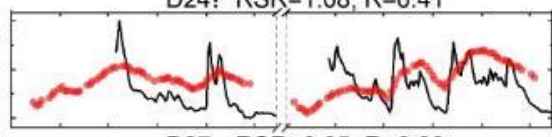

D27: $R S R=0.95, R=0.39$

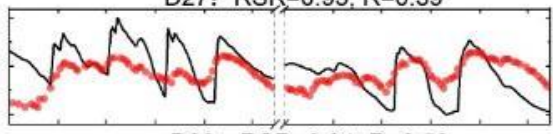

D30: $R S R=0.94, R=0.53$

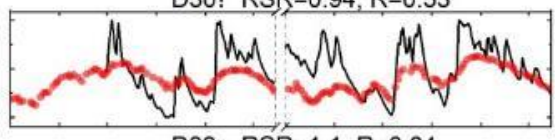

D33: $R S R=1.1, R=0.04$

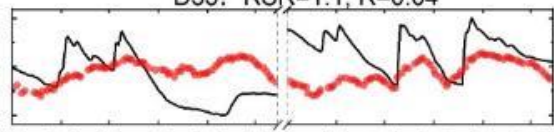

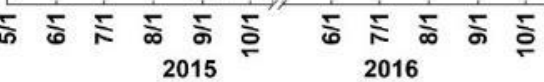

- Observation SWI

$\because$ SMAP estimation SWI

Fig. S4. Comparison of the SMAP estimation and in-situ observation of layer 3 SWI time series during the growing season of $2015-2016$

for the 35 stations. Also with the RMSE and R of the comparisons of the two datasets for each station during the growing season of 20152016. 
$D 1: R S R=1.25, R=-0.31$

0.5 .

0.0 D4: RSR $=1.29, R=0.65$

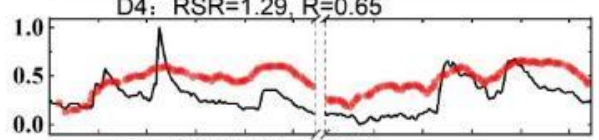

D7: $R S R=1.01, R=0.54$

D10: $R S R=0.8, R=0.66$

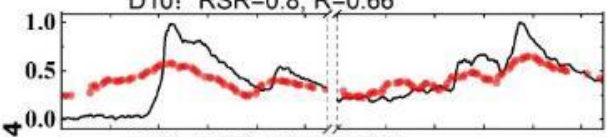

D13: RSR=1.04, R=0.14

要1.0.0

D16: RSR=1.31, R= $=0.24$

ज.

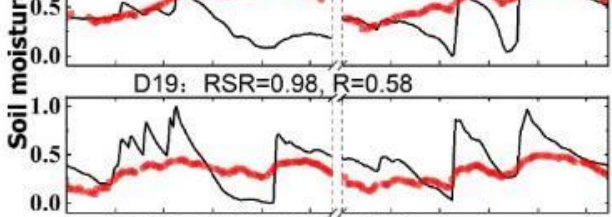

D22: RSR=1.05, R=0.73

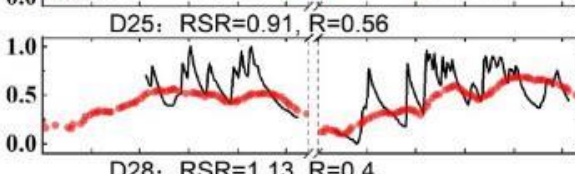

D28: RSR=1.13, $\mathrm{R}=0.4$

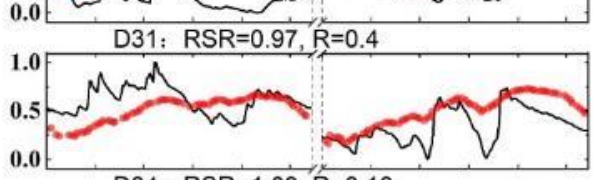

D34: $R S R=1.09, R=0.16$

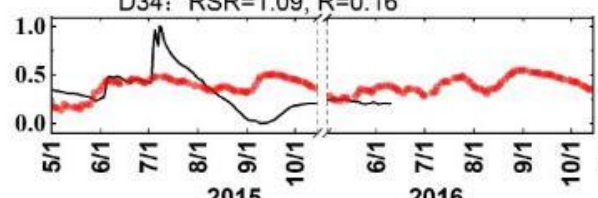

D2: $R S R=1,71, R=0.42$

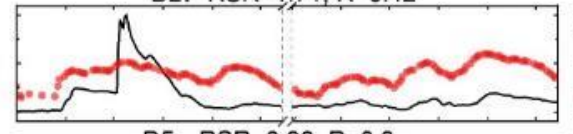

D5: $\mathrm{RSR}=0,96, \mathrm{R}=0.3$

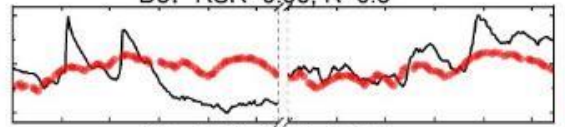

D8: $R S R=1,23, R=0.5$

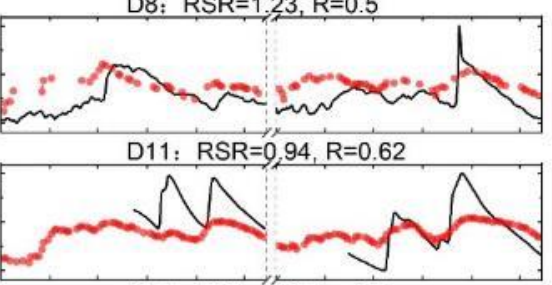

D14: RSR $=1,2, R=-0.5$

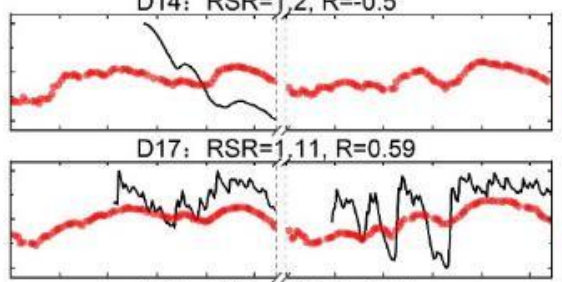

D20: $R S R=1.07, R=0.47$

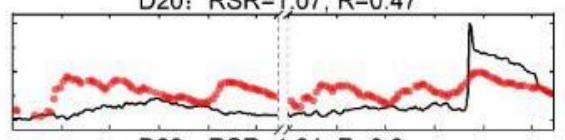

D23: $R S R=1,31, R=0.3$

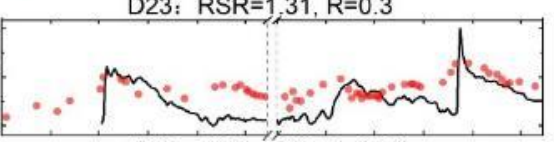

$\mathrm{D} 26$ : $\mathrm{RSR}=1,03, \mathrm{R}=0.29$

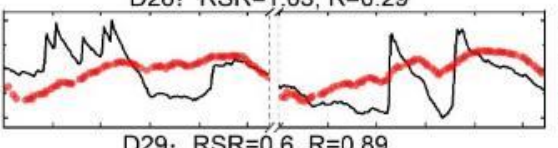

D29: $R S R=0.6, R=0.89$

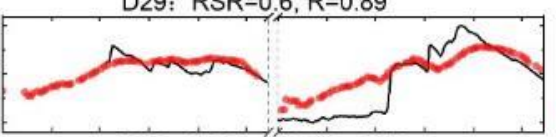

D32: $R S R=1.24, R=-0.45$

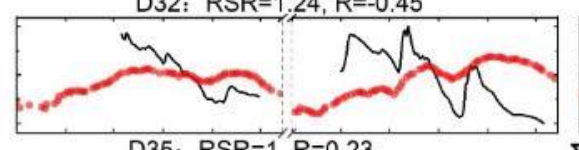

D35: $R S R=1, R=0.23$

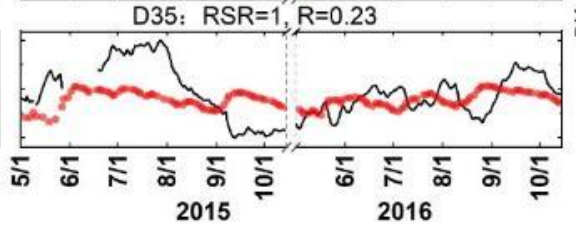

D3: $R S R=1.13, R=-0.14$

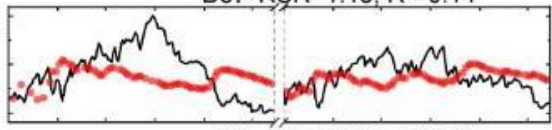

D6: $\mathrm{SSR}=0.94, \mathrm{R}=0.62$

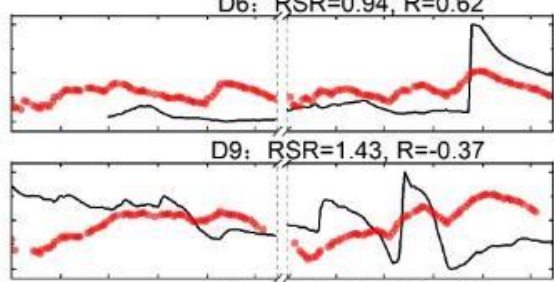

D12: RSR $=0.75, R=0.74$

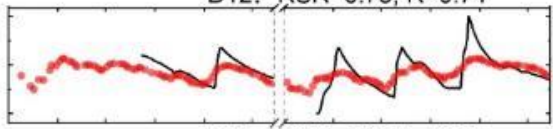

D15: $R S R=1.2, R=-0.26$

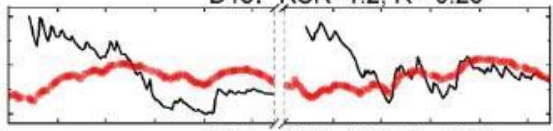

D18: $R S R=1.11, R=-0.18$

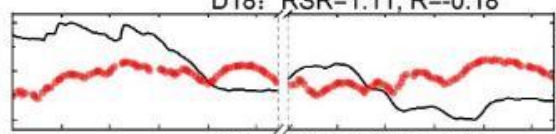

D21: $R S R=0.87, R=0.51$

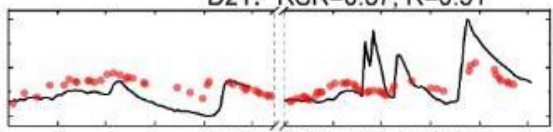

D24: RSR=1.3, R=0.17

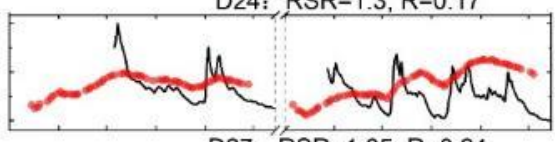

D27: $R S R=1.05, R=0.24$

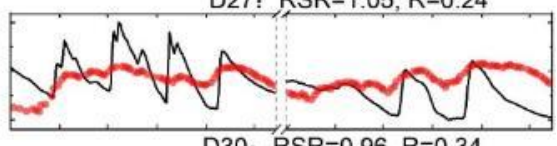

D30: RSR=0.96, $R=0.34$

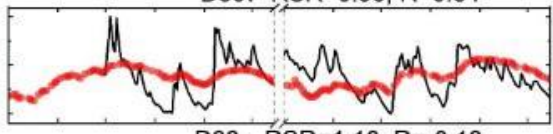

D33: RSR=1.16, R=-0.18

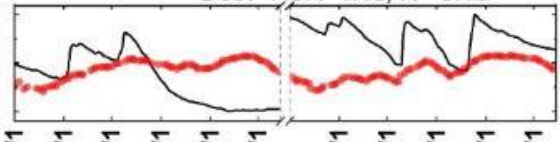

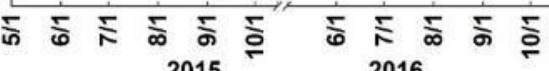
2015

- Observation SWI

$\because$ SMAP estimation SWI

Fig. S5. Comparison of the SMAP estimation and in-situ observation of layer 4 SWI time series during the growing season of $2015-2016$

for the 35 stations. Also with the RMSE and R of the comparisons of the two datasets for each station during the growing season of 2015 2016. 


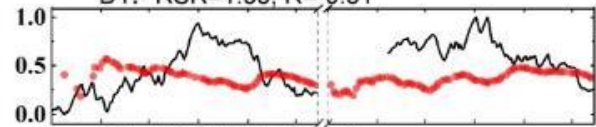

0.0 D4. RSR $=0.78, R=0$
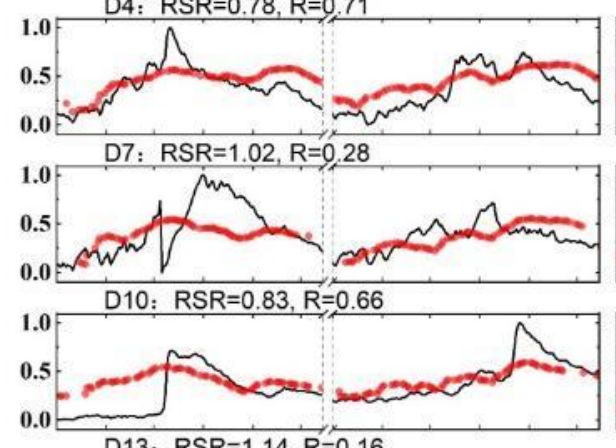

$0.0 \quad \mathrm{D} 13: \mathrm{RSR}=1.14, \mathrm{R}=0.16$
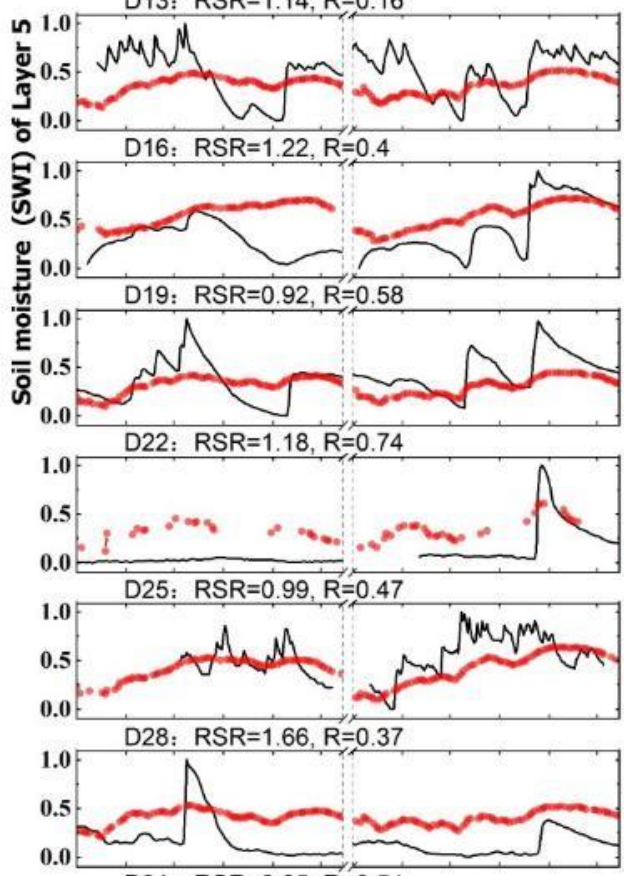

D.0 $31: R S R=0.85, R=0.54$

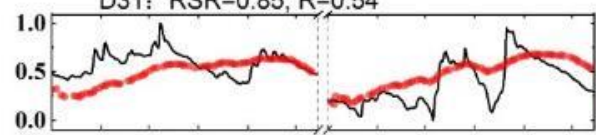

0.0 D34: RSR $=1.13, R=-0.08$

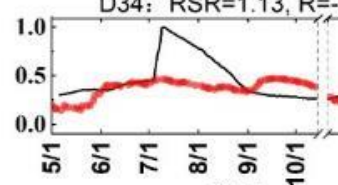
2015

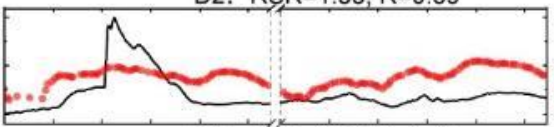

D5: $R S R=1.03, R=0,11$

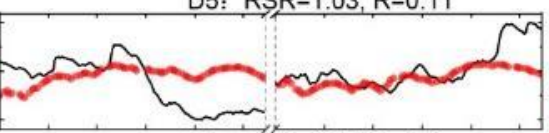

D8: RSR $R=0.97, R=0.3$
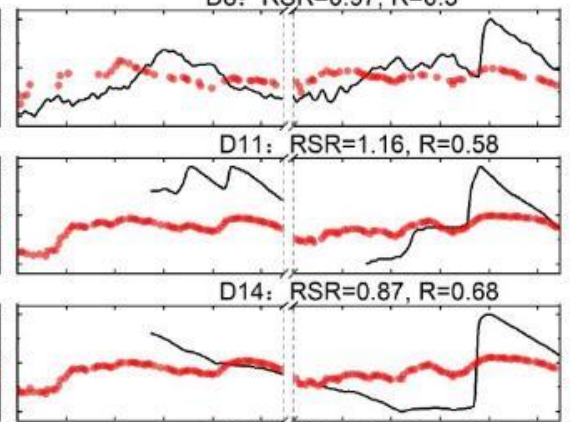

D17: RSR $=1.07, R=0.66$

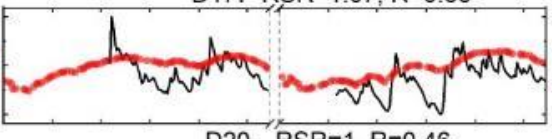

D20: $R S R=1, R=0.46$

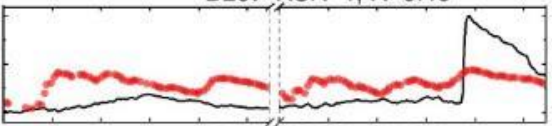

D23: RSR $=0.92, R=0.6$

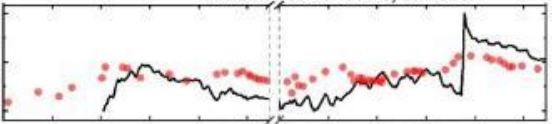

D26: $R S R=1.12, R=0.45$

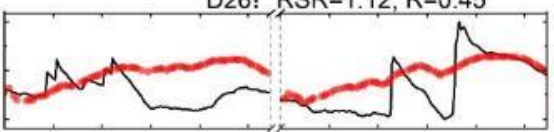

D29: $R S R=0.6, R=0.89$

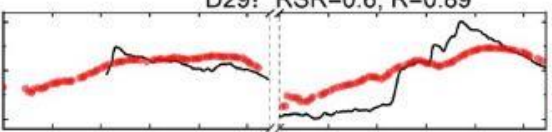

$D 32: \quad R S R=1.29, R=-0.52$

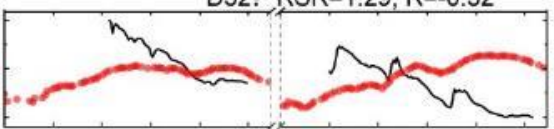

$D 35:, R S R=1.03, R=0.03$

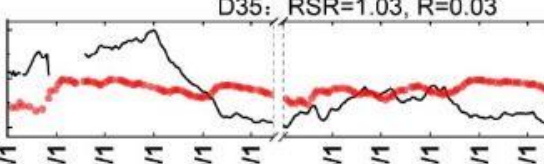

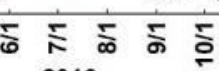

2016

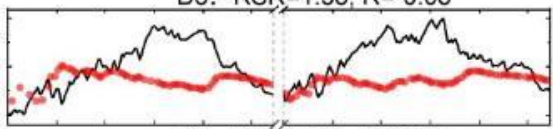

D6: $R S R=1.08, R=0.48$

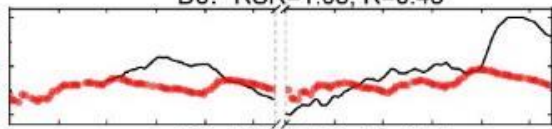

D9: $R S R=1.43, R=-0.55$

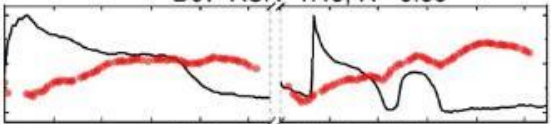

D12: $R S R=0.86, R=0.74$

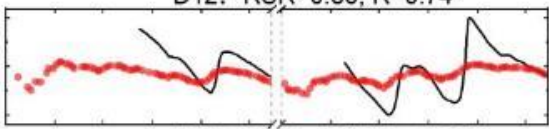

D15: $R S R=1,12, R=-0.15$

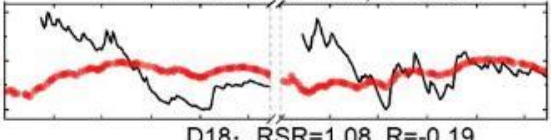

D18: $R S R=1.08, R=-0.19$

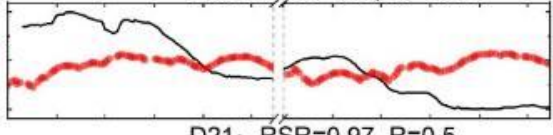

D21: R $S R=0.97, R=0.5$

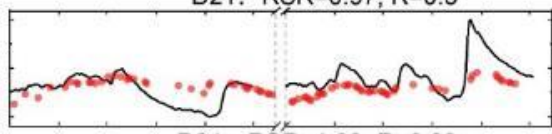

D24: $R S R=1.08, R=0.09$

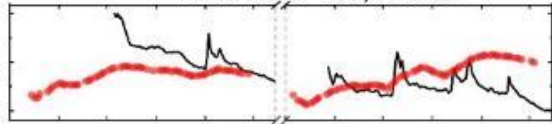

D27: $R S R=1.8, R=-0.06$

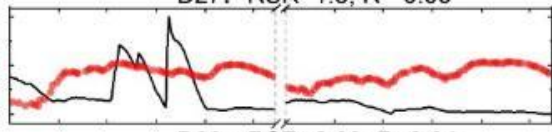

D30: $R S R=2.09, R=0.34$

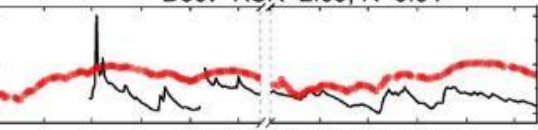

D33: $R$ R $=1.07, R=0.22$

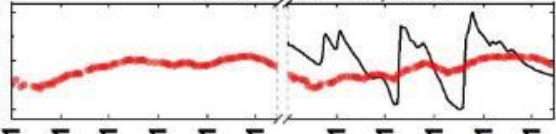

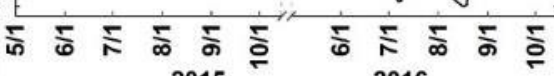
2015 2016

- Observation SWI

$\because$ SMAP estimation SWI

Fig. S6. Comparison of the SMAP estimation and in-situ observation of layer 5 SWI time series during the growing season of $2015-2016$ for the 35 stations. Also with the RMSE and R of the comparisons of the two datasets for each station during the growing season of 2015 2016. 


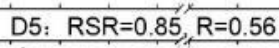

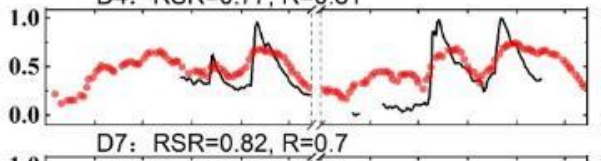

1.0.

$$
\text { inte? }
$$

Andivisarations

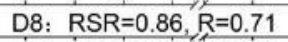

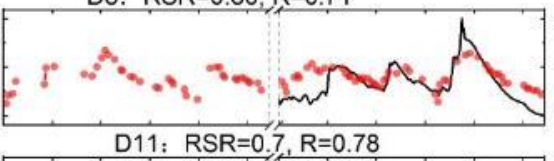

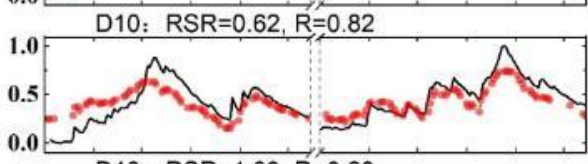

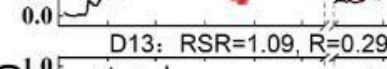

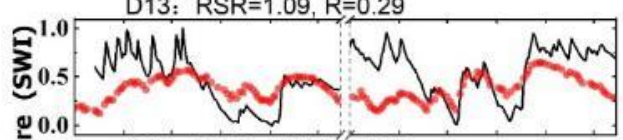

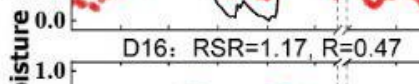

蒙1.0

D19: RSR $=0.85, R=0.79$

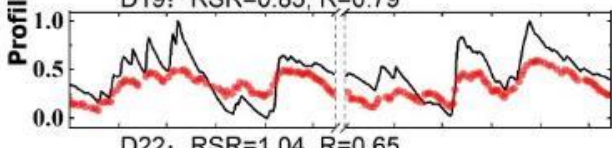

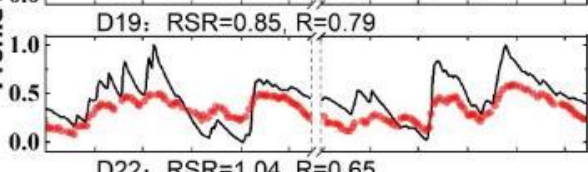

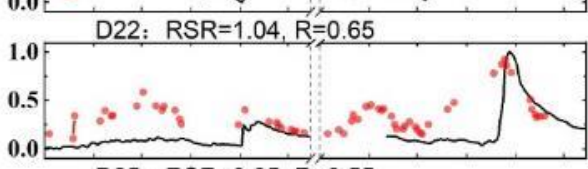

政

(o.

.

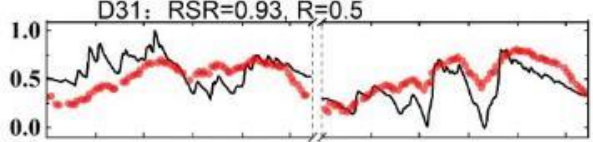

D34: $R S R=0.88, R=0.51$

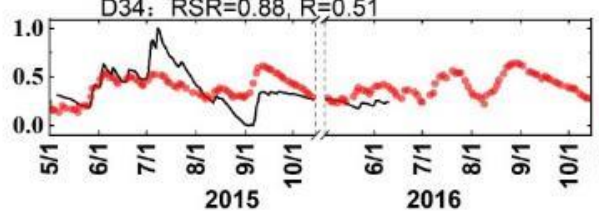

D3: $R S R=0.74, R=0.75$

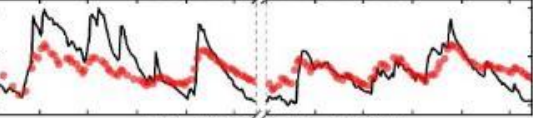

D6: $R S R=0.72, R=0.73$

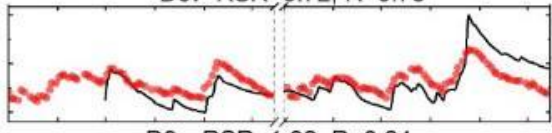

D9: $R S R=1,32, R=0.24$

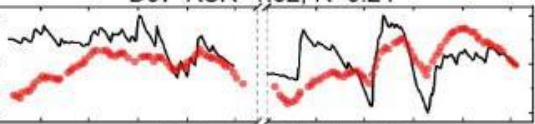

D12: $R S R=0.61, R=0.89$

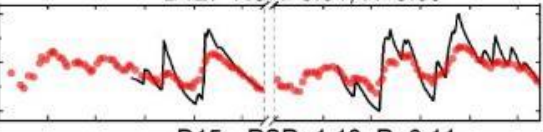

D15: $R S R=1.13, R=0.11$

D14: $R S R=0.96, R=0.36$

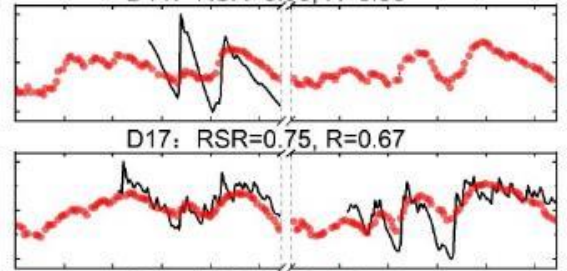

D20: $R S R=0.93, R=0.58$

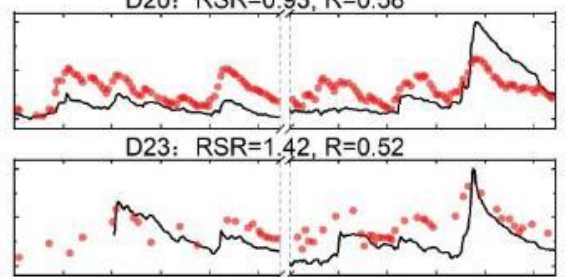

D26: $R S R=0.97, R=0.42$

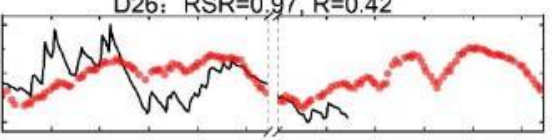

D29: $R S R=0.83, R=0.82$

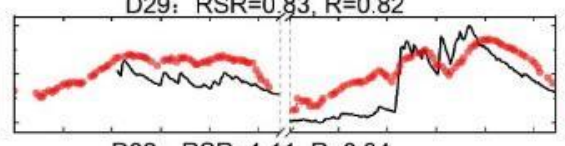

D32: $R S R=1.11, R=0.34$

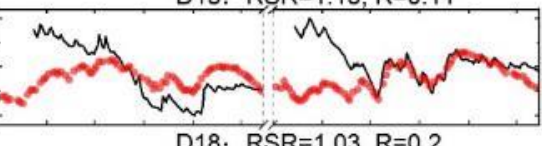

D18: RSR=1.03, $R=0.2$

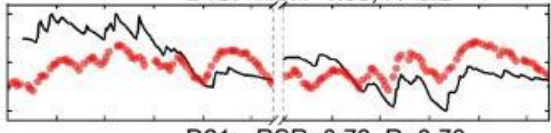

D21: $R S R=0.73, R=0.76$

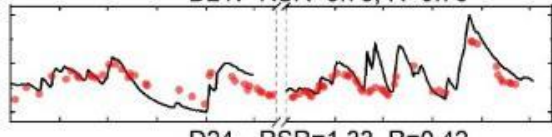

D24: $R S R=1.33, R=0.42$

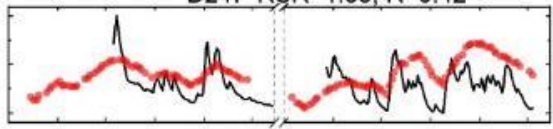

D27: $R S R=0.98, R=0.4$

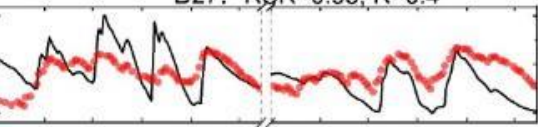

D30: $R S R=0.77, R=0.66$

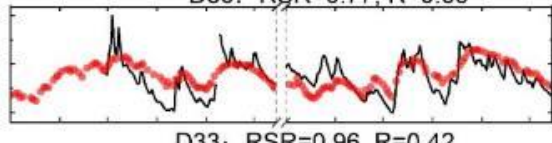

D33: $R S R=0.96, R=0.42$

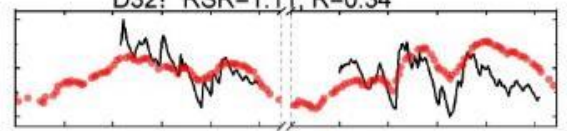

D35: $R S R=0.94, R=0.37$
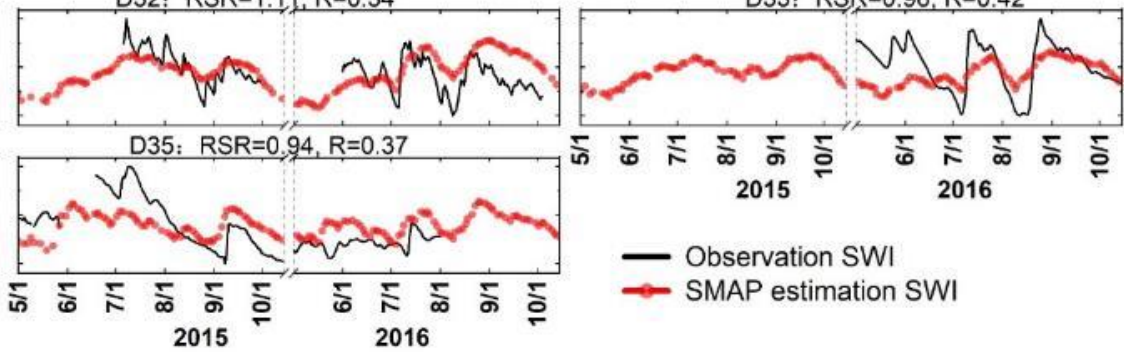

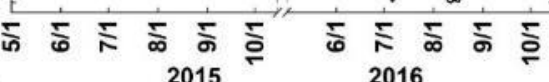

- Observation SWI

- SMAP estimation SWI

Fig. S7. Comparison of the SMAP estimation and in-situ observation of profile SWI time series during the growing season of 2015-2016 for the 35 stations. Also with the RMSE and R of the comparisons of the two datasets for each station during the growing season of $2015-2016$. 

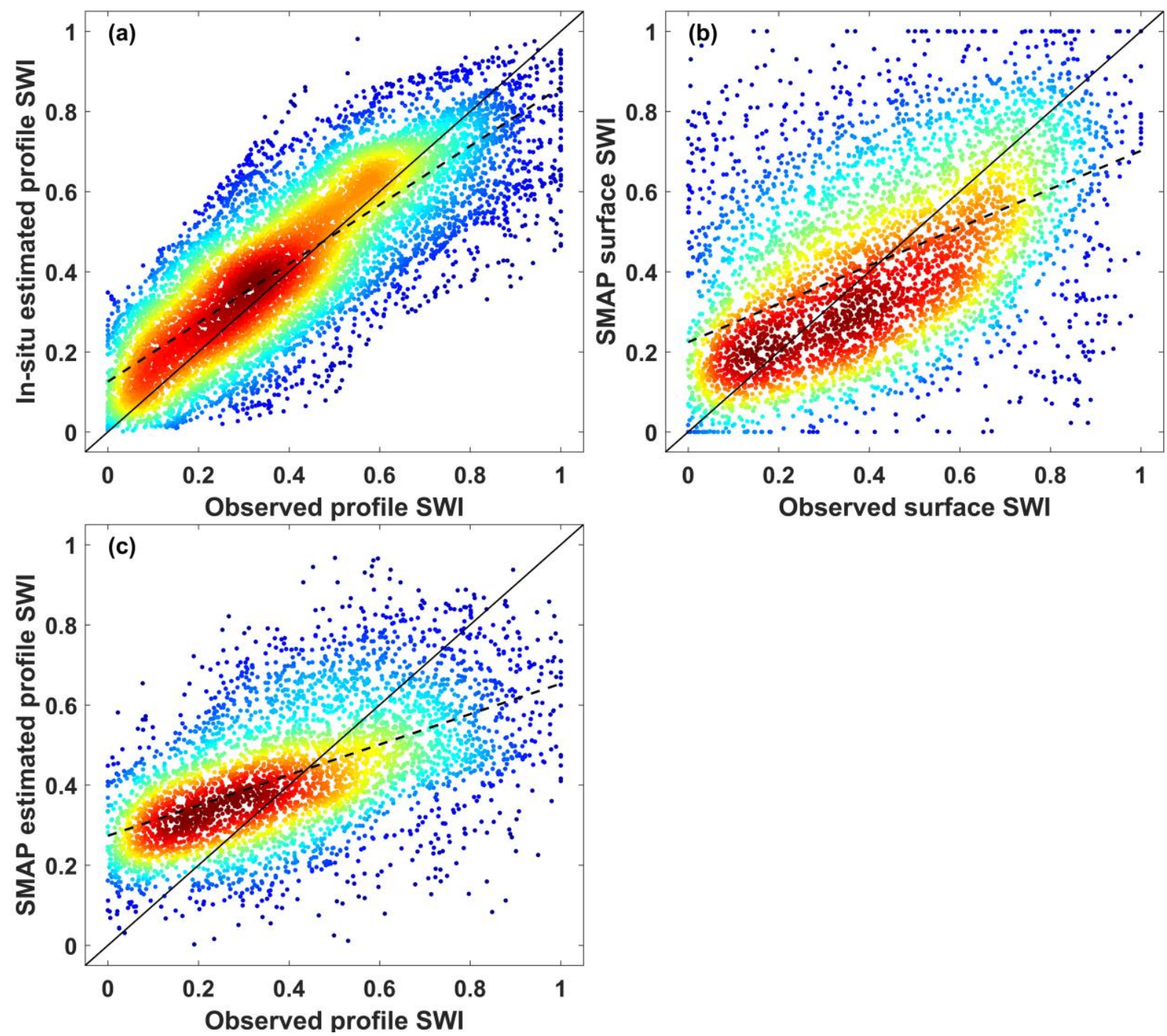

30 Fig. S8. Scatterplot of the comparisons of (a) estimated-observed profile SWI, (b) SMAP-observed surface SWI, and (c) SMAP-observed profile SWI for the 35 stations during the growing seasons of 2015-2016. 


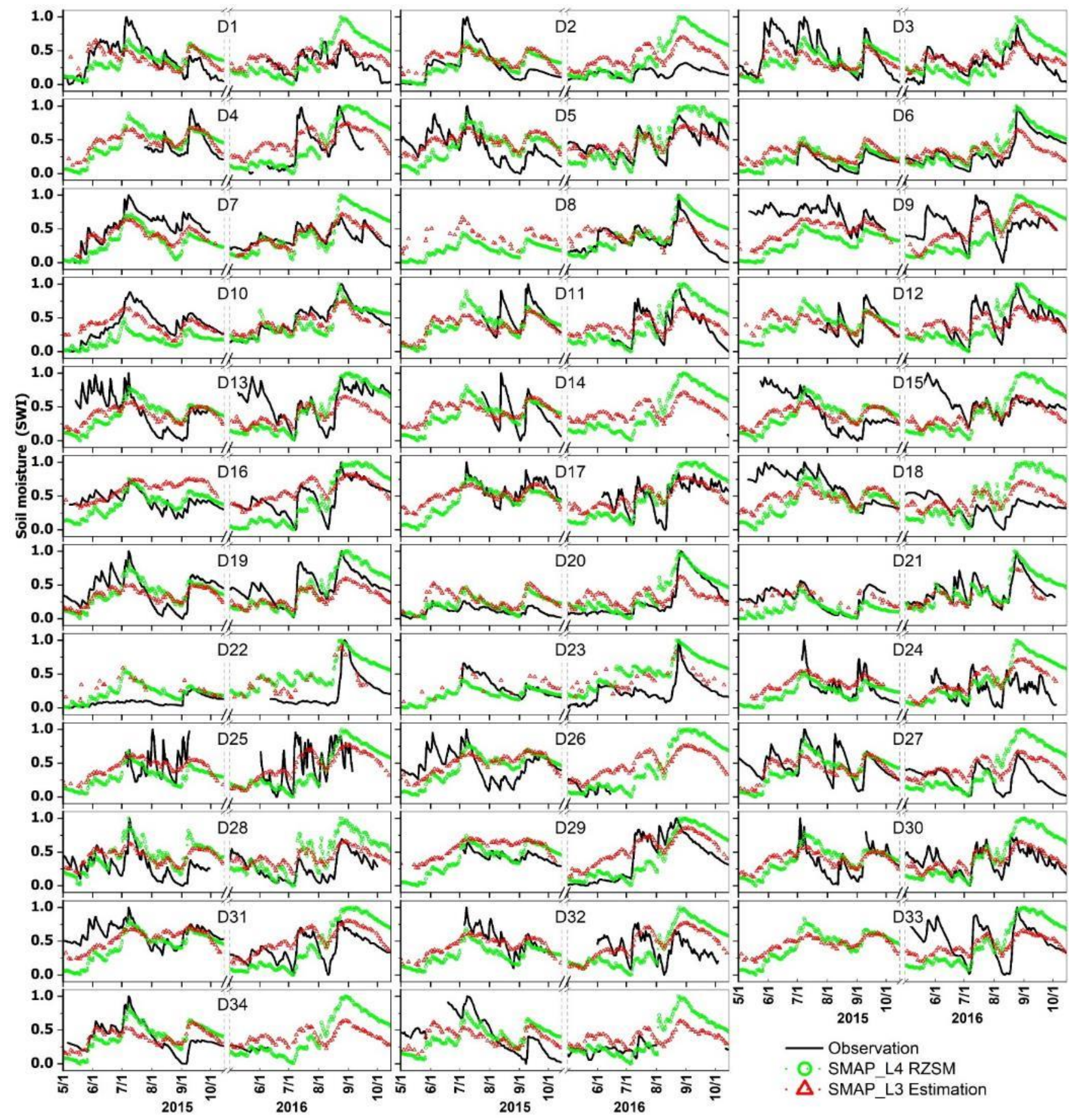

Fig. S9. Comparison of the SMAP_L3 estimated PSWI, SMAP_L4 PSWI and in-situ observation of PSWI time series during the growing season of 2015-2016 for the 35 stations. 
(a)
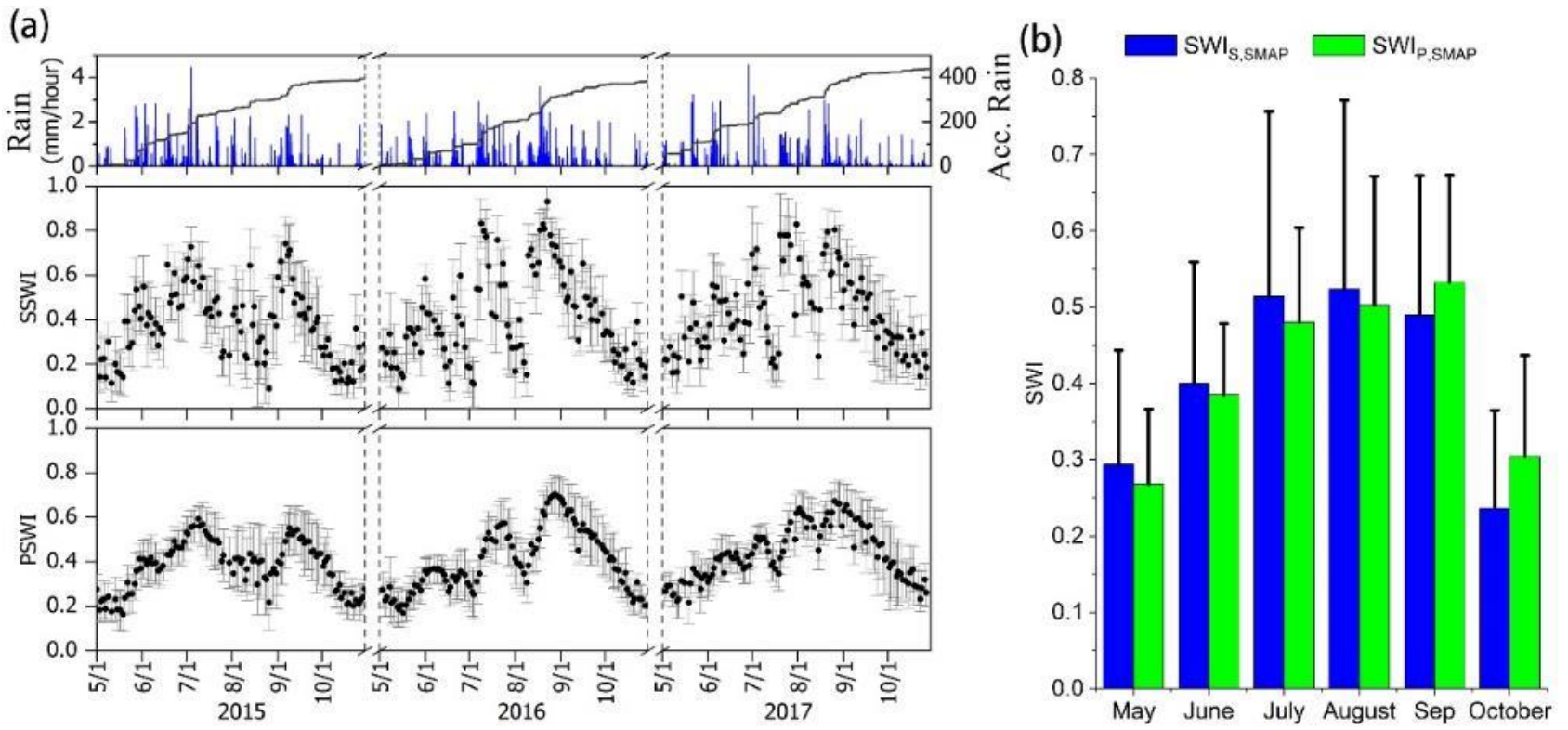

Fig. S10. (a) the temporal variation of precipitation, SSWI and PSWI, and (b) the comparison (bar represents the mean value and error bar means the standard deviation) of the monthly SSWI and PSWI during the growing seasons of 2015-2017. 